\title{
HARMONIC MORPHISMS AND SUBMANIFOLDS WITH CONFORMAL SECOND FUNDAMENTAL FORMS*
}

\author{
XIAOHUAN MO \\ LMAM School of Mathematical Sciences, Peking University, Beijing 100871, China \\ e-mail:moxh@pku.edu.cn
}

(Received 19 November, 2001; accepted 30 May, 2002)

\begin{abstract}
We show that surfaces such that the natural projections of the unit normal bundles are harmonic morphisms are composed of minimal points and totally umbilical points. As its application, we find a harmonic map from the torus to the complex quadric in $C P^{3}$ such that the projection map of the associated sphere bundle constructed by Gudmundsson is not a harmonic morphism. This contrasts sharply with the situation for holomorphic maps. We also establish sufficient conditions for reducing the codimension of an isometric immersion with conformal second fundamental form.
\end{abstract}

2000 Mathematics Subject Classification. 53C12, 53C43, 58E20.

1. Introduction. A class of harmonic maps with a strong geometric flavour is the class of harmonic morphisms. Harmonic morphisms between Riemannian manifolds are solutions to an over-determined non-linear system of partial differential equtions. For this reason they have no general existence theory, not even locally.

For any holomorphic map $\xi: M \rightarrow Q_{n}$ from a Riemannian surface $M$ to an $n$ dimensional complex quadric $Q_{n}$, Gudmundsson constructed in [9] (cf.(4.1)) a sphere bundle $M_{\xi}$ over $M$ with projection map that is a harmonic morphism with totally geodesic fibres; this was later generalized by Aprodu and Aprodu ([1]). Eells and Sampson proved in [6] that any holomorphic map between Kaehler manifolds is harmonic. It is natural to study the following more general situation: Given a harmonic map $\xi: M \rightarrow Q_{n}$ into the complex quadric in $C P^{n+1}$, is the natural projection of $M_{\xi}$ a harmonic morphism?

Let $\phi: M \rightarrow R^{n+2}$ be an isometric immersion with $n \geq 2$. Then its unit normal bundle $T M_{1}^{\perp}$ is exactly the same as $M_{\xi}$ where $\xi$ is the Gauss map of $\phi$. As a natural extension of a result in [9], Gudmundsson and the author proved in [11] that the projection map of the unit normal bundle of a minimal or totally umbilical surface in a Riemannian manifold is a harmonic morphism with totally geodesic fibres (see Proposition 4.2).

In this paper we show that the converse holds, i.e., for any immersed surface in a Riemannian manifold, if the projection map of the unit normal bundle is a harmonic morphism, then the surface can be split into two parts, one is minimal and the other is totally umbilical (see Theorem 5.1).

$\bar{*}$ This work is supported by the National Natural Science Foundation of China 10171002. 
Then we apply Ruh-Vilms' theorem and construct a harmonic map $\xi$ from the torus $S^{1}\left(\sqrt{\frac{1}{2}}\right) \times S^{1}\left(\sqrt{\frac{1}{2}}\right)$ to $Q_{2}$ such that the projection map $\pi:\left(S^{1}\left(\sqrt{\frac{1}{2}}\right) \times S^{1}\left(\sqrt{\frac{1}{2}}\right)\right)_{\xi} \rightarrow$ $S^{1}\left(\sqrt{\frac{1}{2}}\right) \times S^{1}\left(\sqrt{\frac{1}{2}}\right)$ is not a harmonic morphism which gives a negative answer to the problem mentioned above.

Our approach is to discuss the geometry of submanifolds with conformal second fundamental forms (Definition 3.1). First we describe the structure of a surface with conformal second fundamental form (Proposition 3.3) which will be used in the proof of our main result (Theorem 5.1). Meanwhile Proposition 3.3 means that the dimensions of the first normal spaces for surfaces with conformal second fundamental forms are smaller than in the general case. After noting this interesting fact, we investigate the dimensions of the first normal spaces for submanifolds with conformal second fundamental forms (Proposition 3.7). Finally, using dimensional estimation of the first normal spaces and J. Erbacher's reduction theorem we establish sufficient conditions for reducing the codimension of an isometric immersion with conformal second fundamental form in a space of constant curvature (Proposition 3.8).

2. Submanifolds in a Riemannian manifold. Let $M$ be a submanifold immersed into a Riemannian manifold $(N, h)$ by a mapping $\phi: M \rightarrow N$. We denote the second fundamental form (resp. the mean curvature vector) of $\phi$ by $B$ (resp. $H$ ). $\phi$ is said to have parallel mean curvature if $H$ is parallel with respect to the normal connection. In particular, if $H$ vanishes identically, then $\phi$ is said to be minimal.

Let $\xi$ be a normal vector field of $\phi(M)$. Then we define the quadratic differential form by $B_{\xi}=h(B, \xi)$. If $B_{H}$ is proportional to the induced Riemannian metric $\phi^{*} h:=g$, that is, if $B_{H}=\rho g$, then the immersion $\phi$ is said to be pseudo-umbilical. In particular, if $B_{\xi}$ is proportional to $g$ for all normal vectors $\xi, \phi$ is said to be totally umbilical. It is easy to see that the immersion $\phi$ is totally umbilical if and only if $B(X, Y)=g(X, Y) H$ for all tangent vector $X, Y$ of $\phi(M)$, and any minimal submanifold is pseudo-umbilical.

REMARK 2.1. Let $S^{q}(r):=\left\{x \in R^{q+1},\|x\|=r\right\}$ be an Euclidean sphere in an Euclidean space $R^{q+1}$. The inclusion map $i: S^{q}(r) \rightarrow R^{q+1}$ is totally umbilical. Minimal submanifold in Euclidean spheres are submanifolds with parallel mean curvature in Euclidean spaces (see[12], page 167, Remark 2).

Let $\phi:(M, g) \rightarrow(N, h)$ be an isometric immersion and $\xi$ a normal vector field of $\phi(M)$. We denote the shape operator in the direction $\xi$ by $A_{\xi}$, i.e.,

$$
g\left(A_{\xi} X, Y\right)=B_{\xi}(X, Y) \quad X, Y \in \Gamma(T M) .
$$

A simple calculation yields

$$
\operatorname{tr} A_{\xi}=\operatorname{tr} B_{\xi}=m h(H, \xi)
$$

where $m=\operatorname{dim} M$. The following facts will be used in the next section.

LeMma 2.2. Let $\phi:(M, g) \rightarrow(N, h)$ be an isometric immersion.

(1) Let $\xi$ be a normal vector field which is orthogonal to $H$. Then $\operatorname{tr} A_{\xi}=0$;

(2) $\phi$ is a minimal immersion if and only if $\operatorname{tr} A_{H}=0$;

(3) $\phi$ is a pseudo-umbilical immersion if and only if $A_{H}$ is proportional to the identity; 
(4) $\phi$ is a totally umbilical immersion if and only if $A_{\xi}$ is proportional to the identity for all normal vector fields $\xi$;

(5) Suppose that $\phi$ is pseudo-umbilical. Then $\phi$ is totally umbilical if and only if $A_{\xi}=0$ for all normal vector fields $\xi$ which are orthogonal to $H$.

We omit the proof as it is just a direct calculations using (2.1) and (2.2).

3. Isometric immersions with conformal second fundamental form. As a natural generalization of totally umbilical submanifold, we introduce the following (see [11]):

Definition 3.1. Let $(M, g)$ and $(N, h)$ be Riemannian manifolds, and $\phi: M \rightarrow N$ an isometric immersion. The second fundamental form of $\phi$ is said to be conformal if the squares of all the corresponding shape operators along $\phi$ are proportional to the identity.

Since the shape operator $A_{\xi}$ is symmetric for any normal vector field $\xi$, it follows that the condition that $A_{\xi}^{2}$ be proportional to the identity is equivalent to $\left\langle A_{\xi} X, A_{\xi} X\right\rangle=$ $\lambda\langle X, X\rangle$ for all tangent vector fields $X$ and some function $\lambda$, thus explaining why the second fundamental form is called 'conformal'.

EXAMPLE 3.2. Any 2-dimensional minimal surface in a Riemannian manifold has conformal second fundamental form (cf. [11]).

Conversely, we shall prove following

Proposition 3.3. Let $M$ be an immersed surface in a Riemannian manifold $(N, h)$. Then $M$ has conformal second fundamental form if and only if $M=M_{1} \cup M_{2}$ where $M_{1}$ is minimal and $M_{2}$ is totally umbilical. In particular $M$ is a pseudo-umbilical surface.

We require the following result, the proof of which is omitted.

LeMma 3.4. Let $A$ be a real and symmetric $2 \times 2$ matrix. Then $A^{2}$ is proportional to the identity matrix if and only if either the trace of $A$ vanishes or $A$ is proportional to the identity matrix.

Proof. Sufficiency is an immediate consequence of Theorem 4.5 and Example 4.6 in [11]. Let $x \in M$ and $\left\{e_{1}, e_{2}\right\}$ an orthogonal basis of $T_{x} M$. Let $\xi$ be a normal vector field of $\phi(M)$. Denote the matrix of $\left(A_{\xi}\right)_{x}: T_{x} M \rightarrow T_{x} M$ with respect to $\left\{e_{1}, e_{2}\right\}$ by $\tilde{A_{\xi}}$. Then the conformality of second fundamental form implies that $\tilde{A}_{\xi}^{2}$ is proportional to the identity matrix. We work at a non-minimal point. Then (2) in Lemma 2.2 implies that $\operatorname{tr} \tilde{A_{H}} \neq 0$. Together with Lemma 3.4 we have

$$
\tilde{A_{H}} \propto \text { identity matrix. }
$$

It follows from (3) of Lemma 2.2 that $\phi$ is pseudo-umbilical. Consider a normal vector field $\xi$ which is orthogonal to $H$. (1) of Lemma 2.2 tells us that

$$
\operatorname{tr} \tilde{A_{\xi}}=0 .
$$

At $x$, we set $\eta=\frac{H}{\|H\|} \cos \theta+\xi \sin \theta$ where $\theta=$ constant $\in\left(0, \frac{\pi}{2}\right)$. Then (2.1) yields

$$
\tilde{A_{\eta}}=\frac{\cos \theta}{\|H\|} \tilde{A_{H}}+\sin \theta \tilde{A_{\xi}} .
$$


Together with (2.2) and (3.2) we get

$$
\begin{aligned}
\operatorname{tr} \tilde{A_{\eta}} & =\frac{\cos \theta}{\|H\|} \operatorname{tr} \tilde{A_{H}}+\sin \theta \operatorname{tr} \tilde{A_{\xi}} \\
& =2 \cos \theta\|H\| \neq 0 .
\end{aligned}
$$

By the conformality of the second fundamental form of $\phi$ and Lemma 3.4 we get

$$
\tilde{A_{\eta}} \propto \text { identity matrix. }
$$

On the other hand, (3.3) implies that

$$
\tilde{A_{\xi}}=\tilde{A_{\eta}} \csc \theta-\tilde{A_{H}} \frac{c t_{g} \theta}{\|H\|}
$$

from which, together with (3.1) and (3.4), it follows that $\tilde{A_{\xi}}$ is proportional to the identity matrix. Together with (3.2) we have $\tilde{A_{\xi}}=0$. From (5) of Lemma 2.2, $x$ is a totally umbilical point of $\phi$.

By using Obata's result ([14]), we have following

COROLlary 3.5. Suppose that a surface $M$ is isometrically immersed into a space form with conformal second fundamental form. Then the Gauss map is conformal.

Let $M$ be an immersed surface in a Riemannian manifold $(N, h)$ with conformal second fundamental form. By Proposition 3.3, $\mathrm{M}$ is composed of minimal points and totally umbilical points. It is an elementary observation that at a minimal (resp. totally umbilical) point the dimension of the image of the second fundamental form of $M$ is at most two (resp. one) $([\mathbf{1 1}, \mathbf{1 6}])$. We have following

COROLlARY 3.6. Let $M$ be a surface in a Riemannian manifold $(N, h)$ with conformal second fundamental form. Then at each point the dimension of the first normal space, i.e. the image of the second fundamental form of $M$, is at most two.

In the general case, we have

Proposition 3.7. Let $M$ be a submanifold in a Riemannian manifold $(N, h)$ with conformal second fundamental form. If the dimension of $M$ is odd, or if $N$ has constant sectional curvature and the unit mean curvature vector field is parallel in the normal bundle, then at each point the dimension of the first normal space is at most one.

Proof. Let $\xi$ be a normal vector field which is orthogonal to $H$. Then $\operatorname{tr} A_{\xi}=0$ (cf. Lemma 2.2). Choose an orthonormal frame on $M$ such that the shape operator in the direction $\xi$ is diagonalized, that is,

$$
\tilde{A_{\xi}}=\operatorname{diag}\left(\lambda_{1}, \ldots, \lambda_{m}\right)
$$

where $m=\operatorname{dim} M$. By the conformality of the second fundamental form of $M$, we have

$$
\lambda_{1}^{2}=\cdots=\lambda_{n}^{2}=\mu^{2}
$$

where $\mu$ is a locally non-negative function. If $\tilde{A_{\xi}}=\mu I$, then $\tilde{A_{\xi}}=0 \operatorname{using} \operatorname{tr} A_{\xi}=0$ where $I$ denotes identity matrix. Otherwise we can assume

$$
\lambda_{1}=\cdots=\lambda_{p}=-\lambda_{p+1}=\cdots=-\lambda_{m}=\mu .
$$


Then

$$
0=\operatorname{tr} \tilde{A_{\xi}}=(2 p-m) \mu .
$$

If $m$ is odd, then we have $\mu=0$ which implies that $A_{\xi}=0$. Together with (2.1) we have

$$
\|H\|^{2} B=B_{H} H .
$$

Hence the first normal space is generated by $H$. In the general case, using the conformality of the second fundamental form of $M$ we have

$$
\begin{gathered}
\tilde{A_{\xi}} \tilde{A_{H}}+\tilde{A_{H}} \tilde{A_{\xi}}=\tilde{A_{\xi+H}^{2}}-\tilde{A_{\xi}^{2}}-\tilde{A_{H}^{2}}=\tau_{\xi} I, \\
\tilde{A_{H^{2}}}=\sigma I .
\end{gathered}
$$

If $(N, h)$ has constant sectional curvature and the unit mean curvature vector field is parallel in the normal bundle, then $[17,19]$

$$
\tilde{A_{\xi}} \tilde{A_{H}}=\tilde{A_{H}} \tilde{A_{\xi}} \text {. }
$$

Substitute (3.6) into (3.4) we have

$$
\tilde{A_{\xi}} \tilde{A_{H}}=\frac{1}{2} \tau_{\xi} I
$$

The existence of the unit mean curvature vector implies that $\|H\| \neq 0$. Using (2.2) we get $\operatorname{tr} \tilde{A_{H}} \neq 0$. Together with (3.5) we have $\operatorname{det} \tilde{A_{H}} \neq 0$. Thus from (3.5) and (3.7) we see

$$
\tilde{A_{\xi}}=\frac{1}{2} \tau_{\xi} \tilde{A_{H}^{-1}}=\frac{\tau_{\xi}}{2 \sigma} \tilde{A_{H}}
$$

Combine with (2.1) we have

$$
B=\Sigma_{\alpha} B_{e_{\alpha}} e_{\alpha}=\Sigma_{\alpha} \frac{\tau_{e_{\alpha}}}{2 \sigma} B_{H} e_{\alpha}=\frac{1}{2 \sigma} B_{H} V
$$

where

$$
V:=\Sigma_{\alpha} \tau_{e_{\alpha}} e_{\alpha}
$$

and $\left\{e_{\alpha}\right\}$ an orthonormal frame of the normal bundle of $M$.

We now use Proposition 3.7 to show the following reduction of the codimension of an isometric immersion with conformal second fundamental form. We assume that $M$ is a connected manifold.

Proposition 3.8. Let $N$ be a space of constant curvature and $M$ an $m$-dimensional submanifold of $N$ with conformal second fundamental form. Suppose that $m$ is odd and $M$ has parallel mean curvature, or $M$ has flat normal bundle and the first normal spaces of $M$ have constant dimension. Then either

(1) $M$ is totally geodesic, i.e. $B \equiv 0$, or

(2) $M$ lies in an $(m+1)$-dimensional totally geodesic submanifold with conformal second fundamental form. 
Proof. Suppose that $\operatorname{dim} M$ is odd. From the metric-compatibility of Levi-Civita connection and the connectedness of $M$, it is easy to see that, if $M$ has parallel mean curvature, then either

(i) $M$ is minimal or

(ii) $\|H\|$ is a non-zero constant and $M$ has parallel unit mean curvature vector field.

Suppose that $M$ is minimal, then as in the proof in Proposition 3.7 we have $A_{\xi}=0$ for all normal vector field $\xi$. Hence $M$ is a totally geodesic submanifold from (2.1). Suppose instead that $\|H\|$ is non-zero constant and $M$ has parallel unit mean curvature vector field. Then, from the proof of Proposition 3.7, the image of the second fundamental form of $M$ is generated by the mean curvature vector field. Using J. Erbacher's reduction theorem [7], we have that $M$ is a hypersurface of a totally geodesic submanifold, denoted by $P$, of $N$. Moreover the shape operator of $M$ in $P$ along a normal vector field $\xi$ is exactly the same as the shape operator $M$ in $N$ along $\xi$. Hence $M$ is a hypersurface in $P$ with conformal second fundamental form. Next, suppose that $M$ has flat normal bundle and the dimensions of the first normal spaces are constant. If $M$ is not totally geodesic, then $A_{\xi} \neq 0$ for some normal vector field $\xi$. Since $N$ has constant sectional curvature, the normal bundle of $M$ is flat if and only if all the shape operators can be diagonalized simultaneously [17]. Using a similar argument to that the proof of Proposition 3.7 we have all first normal spaces of $M$ form a rank one vector bundle. The flatness of the normal bundle implies that this bundle is parallel with respect to the normal connection. Again using J. Erbacher's reduction theorem we get (2).

4. Sphere bundles over surfaces and harmonic maps. Let $C P^{n+1}$ be the $(n+1)$ dimensional complex projective space with the Fubini-Study metric and $Q_{n}\left(\subset C P^{n+1}\right)$ be the $n$-dimensional complex quadric. Notice that $Q_{n}$ with induced Kaehler metric is isometric to the real Grassmannian $G_{2}\left(R^{n+2}\right)([17])$. Let $M$ be a surface. Using the standard inner product in $R^{n+2}$, we associate to the map $\xi: M \rightarrow Q_{n}$ the $S^{n-1}$-bundle (cf. [9])

$$
M_{\xi}=\left\{(z, x) \in M \times S^{n+1} \mid \xi(z) \perp x\right\}
$$

with projection map $M_{\xi} \rightarrow M$ given by the restriction of the natural projection. Let $n \geq 2$ and let $\phi: M \rightarrow R^{n+2}$ be an isometric immersion of a surface and $\xi$ its Gauss map. Then the unit normal bundle of $M$

$$
T M_{1}^{\perp}:=\left\{(z, x) \in T M_{1}^{\perp}\|x\|=1\right\}
$$

is the $S^{n-1}$-bundle $M_{\xi}$ associated to $\xi([9,11])$.

Let $P$ and $Q$ be Riemannian manifolds. A map $f: P \rightarrow Q$ is called harmonic if it is a critical point of the energy functional

$$
\frac{1}{2} \int_{D}\|d f\|^{2} d v .
$$

For a detailed account of harmonic maps of a compact domain $D$ see $[\mathbf{4}, \mathbf{5}]$. As an example, for an isometric immersion $\phi: P \rightarrow R^{n+2}$ with parallel mean curvature, the Gauss map of $\phi$ is harmonic [15]. A map $f: P \rightarrow Q$ between Riemannian manifolds is called a harmonic morphism if for any harmonic function $\psi: U \rightarrow \mathrm{R}$ defined on an open subset $U$ of $Q$ with $f^{-1}(U)$ non-empty, $\psi \circ f: f^{-1}(U) \rightarrow \mathrm{R}$ is a harmonic 
function. We refer the reader to [2] for the basic properties of and further references to harmonic morphisms. Recall (cf. $[\mathbf{8}, \mathbf{1 3}]$ ) that a map $f: P \rightarrow Q$ between Riemannian manifolds is a harmonic morphism if and only if it is harmonic and horizontally (weakly) conformal, in the sense that, at any point $p \in P$ not contained in the critical set $C_{f}=\left\{p \in P \mid d f_{p}=0\right\}$ of $f$, the restriction of $d f_{p}$ to the orthogonal complement

$$
\left\{X \in T_{p} P \mid\langle X, Y\rangle=0 \text { for all } Y \in \operatorname{Ker} d f_{p}\right\}
$$

of $\operatorname{Ker} d f_{p}$ is surjective and conformal onto the tangent space $T_{f(p)} Q$. It is easy to see that the class of horizontally (weakly) conformal maps includes Riemannian submersions.

Let $\xi: M \rightarrow Q_{n}$ be a map from a surface $M$. Denote by $i: Q_{n} \rightarrow C P^{n+1}$ the inclusion map. Since $Q_{n}$ is a Kaehler hypersurface of $C P^{n+1}$ it follows that $\xi$ is holomorphic if and only if $i \circ \xi$ is holomorphic. Combining with Proposition 3.1 in [9] we have.

Proposition 4.1. Let $\xi: M \rightarrow Q_{n}$ be a holomorphic curve and $M_{\xi}$ its associated sphere bundle. Then the projection map from $M_{\xi}$ to $M$ is a harmonic morphism.

Proposition 4.2. ([11]) Let $(M, g)$ be a minimal or totally umbilical surface in a Riemannian manifold $(N, h)$ and $T M_{1}^{\perp}$ be the unit normal bundle of $M$ in $(N, h)$. Then the projection map

$$
\pi:\left(T M_{1}^{\perp}, d s^{2}\right) \rightarrow(M, g)
$$

is a harmonic morphism with totally geodesic fibre. Here $d s^{2}$ denotes the restriction of the Sasaki metric on $T N$ to the submanifold $T M_{1}^{\perp}$.

For more interesting examples of harmonic morphisms we refer the reader to [10] on the World Wide Web.

5. Main result and its application. In this section, we prove the main result of this note (Theorem 5.1) which completes Proposition 4.2. As an application, we give a negative answer to the problem described in the introduction.

THEOREM 5.1. Let $\phi:(M, g) \rightarrow(N, h)$ be an isometric immersion from a surface $M$ and $\left(T M_{1}^{\perp}, d s^{2}\right)$ its unit normal bundle where $d s^{2}$ is the restriction of the Sasaki metric on $T N$ to $T M_{1}^{\perp}$. Then the projection map $\pi:\left(T M_{1}^{\perp}, d s^{2}\right) \rightarrow(M, g)$ is a harmonic morphism if and only if $M=M_{1} \cup M_{2}$ where $M_{1}$ is minimal and $M_{2}$ is totally umbilic, i.e. $M$ is composed of minimal points and totally umbilical points.

Proof. By Proposition 3.3 it is sufficient to show that $\pi$ is a harmonic morphism if and only if $\phi$ has a conformal second fundamental form. Suppose that $\pi$ is a harmonic morphism. By using Proposition 4.2 in [11] we have $\phi$ has a conformal second fundamental form. The converse is an immediate consequence of Proposition 4.4 in [11] since dimension of $M$ is two.

An important application of Theorem 5.1 is to give a negative answer of the interesting problem mentioned in the introduction, as follows.

Let $S^{q}(r)$ denote a $q$-dimensional sphere in $R^{q+1}$ with radius $r$. Let $m$ and $n$ be positive integers such that $m<n$ and let $M_{m, n-m}=S^{m}\left(\sqrt{\frac{m}{n}}\right) \times S^{n-m}\left(\sqrt{\frac{n-m}{n}}\right)$. The image of the canonical embedding $M_{m, n-m} \rightarrow R^{n+2}$ lies in the unit sphere $S^{n+1}(1)$. We call $M_{m, n-m}\left(\subset S^{n+1}(1)\right)$ a Clifford hypersurface. We have the following. 
Proposition 5.2. Let $M$ be a Clifford hypersurface in $S^{n+1}(1) \subset R^{n+2}$. As a submanifold in $R^{n+2}$ of codimension two, then

(i) $M$ is a pseudo-umbilical submanifold;

(ii) $M$ has parallel mean curvature;

(iii) There are neither totally umbilical points nor minimal points on $M$.

Proof. Let $e_{0}, e_{1}, \ldots, e_{m}$ be an orthonormal frame field for $R^{m+1}$ such that $e_{0}$ is normal to $S^{m}\left(\sqrt{\frac{m}{n}}\right)$. Similarly, for $S^{n-m}\left(\sqrt{\frac{n-m}{n}}\right)$ in $R^{n-m+1}$, we choose an orthonormal frame field $e_{m+1}, \ldots, e_{n+1}$ such that $e_{n+1}$ is normal to $S^{n-m}\left(\sqrt{\frac{n-m}{n}}\right)$. Put

$$
\xi=\sqrt{\frac{m}{n}} e_{0}+\sqrt{\frac{n-m}{n}} e_{n+1}, \quad \eta=\sqrt{\frac{n-m}{n}} e_{0}-\sqrt{\frac{m}{n}} e_{n+1} .
$$

Then $\xi$ is normal to $S^{n+1}(1)$ and $\eta$ is normal to $M_{m, n-m}$. On $M_{m, n-m},\{\xi, \eta\}$ is an orthonormal normal frame. By the total umbilicity of $S^{m}\left(\sqrt{\frac{m}{n}}\right), S^{n-m}\left(\sqrt{\frac{n-m}{n}}\right)$ and $S^{n+1}(1)$, we have, with respect to $\left\{e_{1}, \ldots, e_{n}\right\}$ (cf.[3]),

$$
\tilde{A_{\eta}}=\operatorname{diag}(\underbrace{\lambda, \ldots, \lambda}_{m}, \underbrace{\mu, \ldots, \mu}_{n-m}), \quad \tilde{A_{\xi}}=\text { identity matrix }
$$

where $\lambda=\sqrt{(n-m) / m}$ and $\mu=-\sqrt{m /(n-m)}$. So $\operatorname{tr} \tilde{A_{\eta}}=0$. Combining this with (2.2) shows that $\xi$ is the mean curvature vector field of submanifold $M_{m, n-m} \subset R^{n+2}$. Now (ii) is an immediate consequence of $\operatorname{tr} \tilde{A_{\eta}}=0$ and Remark 2.1. Part (i) follows from (3) of Lemma 2.2. Using (2) and (5) of Lemma 2.2 and $\operatorname{tr} \tilde{A_{\xi}} \neq 0, \tilde{A_{\eta}} \neq 0$ we now obtain (iii).

Consider $n=2$ and $m=1$, i.e. $M_{1,1}=S^{1}\left(\sqrt{\frac{1}{2}}\right) \times S^{1}\left(\sqrt{\frac{1}{2}}\right) \rightarrow R^{4}$. We have the following.

COROLlARY 5.3. There is a harmonic map $\xi: M_{1,1}=S^{1}\left(\sqrt{\frac{1}{2}}\right) \times S^{1}\left(\sqrt{\frac{1}{2}}\right) \rightarrow Q_{2}$ such that the projection map $\pi:\left(M_{1,1}\right)_{\xi} \rightarrow M_{1,1}$ is not a harmonic morphism.

Proof. Let $\phi: M_{1,1} \rightarrow R^{4}$ be the inclusion map and $\xi: M_{1,1} \rightarrow Q_{2}$ its Gauss map. Then $\phi$ has parallel mean curvature from Proposition 5.2. Together with Ruh-Vilms' theorem [15] we get $\xi$ is harmonic. Recall that $Q_{2}$ with induced Kaehler metric is isometric to the real Grassmannian $G_{2}\left(R^{4}\right)$ ([18]). Again using Proposition 5.2, $\phi$ has neither totally umbilical points nor minimal points. Theorem 5.1 tells us that the unit normal projection $\pi:\left(T M_{1}^{\perp}, d s^{2}\right) \rightarrow(M, g)$ is not a harmonic morphism. As we mention in section 4 , the unit normal bundle of $M_{1,1}$ is exactly the same as the $S^{1}$ bundle associated to $\xi$. It follows that the projection map $\pi:\left(M_{1,1}\right)_{\xi} \rightarrow M_{1,1}$ is not a harmonic morphism.

ACKNOWLEDGEMENTS. This paper has benefited from my discussions with Sigmundur Gudmundsson. The author would like to express his gratitude to J. C. Wood and E. Loubeau for their valuable comments and the referee for his careful reading of the manuscript and very helpful suggestions. The last version of this note was written during a visit to the Max-Planck Institute for Mathematics, Bonn, and the author would also like to thank Professor Y. Manin and the Institute for their hospitality. 


\section{REFERENCES}

1. M. A. Aprodu and M. Aprodu, Implicity defined harmonic PHH submersions, Manu. Math. 100 (1999), 103-121.

2. P. Baird and J. C. Wood, Harmonic morphisms between Riemannian manifolds, London Mathematical Society Monographs (N.S.) (Oxford University Press), to appear.

3. S. S. Chern, M. do Carmo and S. Kobayashi, Minimal submanifolds on a sphere with second fundamental form of constant length, Shiing-shen Chern Selected Papers, 393-409.

4. J. Eells and L. Lemaire, Selected topics in harmonic maps CBMS Reg. Conf. Ser. N.S. (Amer. Math. Soc., 1983).

5. J. Eells and L. Lemaire, Another report on harmonic maps, Bull. Lond. Math. Soc. 20 (1988), 385-524.

6. J. Eells and J. H. Sampson, Harmonic mappings of Riemannian manifolds, Amer. J. Math. 86 (1964), 109-160.

7. J. Erbacher, Reduction of the codimension of an isometric immersion, J. Diff. Geom. 5 (1971), 333-340.

8. B. Fuglede, Harmonic morphisms between Riemannian manifolds, Ann. Inst. Fourier 28 (1978), 107-144.

9. S. Gudmundsson, Harmonic morphisms as sphere bundles over compact Riemann surfaces, Internat. J. Math. 8 (1997), 421-433.

10. S. Gudmundsson, E. Loubeau, S. Montaldo and M. T. Mustafa, The atlas of harmonic morphisms, http://www.maths.lth.se/matematiklu/personal/sigma/harmonic /atlas.html.

11. S. Gudmundsson and X. Mo, Harmonic morphisms as unit normal bundles of minimal surfaces, Manuscripta Math. 100 (1999), 323-333.

12. D. A. Hoffman, Surfaces of constant mean curvature of constant curvature, J. Diff. Geom. 8 (1973), 161-176.

13. T. Ishihara, A mapping of Riemannian manifolds which preserves harmonic functions, J. Math. Kyoto. Univ. 19 (1979), 215-229.

14. M. Obata, The Gauss map of immersions of Riemannian manifolds in spaces of constant curvature. II, J. Diff. Geom. 2 (1968), 217-223.

15. E. A. Ruh and J. Vilms, The tension field of the Gauss map, Trans. Amer. Math. Soc. 149 (1970), 569-573.

16. M. Sakaki, Remarks on the rigidity and stablity of minimal submanifolds, Proc. Amer. Math. Soc. 106 (1989), 793-795.

17. C. L. Terng, Submanifolds with flat normal bundle, Math. Ann. 277 (1987), 95-111.

18. J. G. Wolfson, Harmonic maps of the two-sphere into the complex hyperquadric, J. Diff. Geom. 24 (1986), 141-152.

19. S. T. Yau, Submanifolds with constant mean curvature I, Amer. J. Math. 96 (1974), 346-366. 